\title{
The Light Media in the Application of Commercial Space
}

\author{
Xiaoli Sun \\ Rizhao vocational and technical college
}

\begin{abstract}
Keywords: Light coal; Commercial space; Innovative use of
\end{abstract}
\begin{abstract}
With the improving of the quality of people's life level, people for lighting design media design requirements are more strict, a lighting in commercial space has gradually become an indispensable important constituent, is extensively used in business. Therefore, by the lights of the scientific and effective media lighting can play an effective role in the business. In this paper, from the light media to analyze the current problems in the commercial space, and puts forward how to realize the light media application in the commercial space, hope to be able to give the lights of the current media application in commercial provide certain help.
\end{abstract}

\section{Preface}

In recent years, since the light media along with the social progress and improve the level of consumption, the commercial space also pay more attention to the application of light. In real life, business activities are generally become people engaged in social activities and an important place for shopping, sightseeing, become the important means to enhance the competitiveness of business, therefore, we must have to be the overall design of commercial space environment, so as to make the business shoppers can have a good shopping environment and atmosphere. In general, the light media in the commercial use can greatly enhance the activity of the utility, however, from the point of the current situation, a lot of business space using bought high-grade lighting equipment, tend to be less than its business effect, so we need to pay attention to the light media in commercial use of Settings, from commercial space environment according to the overall layout to design the light media.

\section{A lighting in commercial use of the present situation and development trend}

From a lighting technology is mainly used in commercial lighting for the goods to the rise of the enthusiasm for the goods. After years of development, the light media design in the content of the commercial space shows more colorful. In general, commercial lighting design lighting requirements from the start at the beginning of the design can be goods and indoor environment is closely linked, and through the indoor environment to further show the brand image, thus to indoor environment and lighting as well as sales gradually together. At the same time, with the development of green economy and the growing popularity of the concept of green construction, people for green lighting design aspects put forward the more strict requirements.

On the one hand, we can know the lights of the media business for lighting environment beautification, sales have certain help, therefore, we need through the use of reasonable lighting design to gradually perfected and developed further create certain conditions for business development, and with the development of green economy, energy conservation and environmental protection of green lighting design gradually aroused people's concern, so, the respect is designed in light media must pay more attention to saving lighting, such not only can be beneficial to environmental protection, more can improve lighting technology, which can form a comfortable lighting environment of economic security. And skillfully use the new technology and new technique and new products to highlight indoor space environment, so we can see that in the use of commercial space, we not only achieve efficient lighting environment, more light to be able to create a unique and elegant atmosphere, so that they can for the future of commercial activities provide important technical basis. 


\section{Commercial lighting environment design principles}

\section{Business functional design principles}

From the previous lighting design, can be found that previous business pay more attention to practical lighting design in the space, are often ignored the role of lighting design in commercial activities, and the principle of functional lighting design is lighting design is very good for us to solve this problem. Functional design principles are generally in order to be able to better adapt to the design requirements of lighting environment. So that they can achieve for sales people can produce the physical and mental and emotional comfort. To this end, we need for the characteristics of different space environment and product design, and can according to the specific environment appropriate to adjust the brightness of the lighting, in the large space environment, for example, the way we can adopt the top light, and to be able to make sure the softness of lamplight, try to avoid glare. How is the furniture of lighting for large, we can adopt the method of comprehensive illuminate, but can use local highlight, so to some extent can show furniture unique temperament. If it is a window and commodity display, etc., can adopt the method of direct light, the main goal is to be able to highlight the characteristics of goods, to this, in this case, we can ask in the brightness of the lighting design aspects should be strengthened, and can be used to shoot the light of lamps and lanterns, so it can illuminate the whole interior space environment, can be seen from the above a few examples of, seen in the decoration in the commercial space in the space environment to consider not only the expression of light intensity and color and adornment sex, more need to be able to consider to decorate the amount of light. On the one hand, we must not only can improve the space environment of goods from the point of view of the comfort and visible light, etc., but also can be in strict accordance with the visual acuity and light from these aspects, such as the brightness of the and it should be able to with the details of the intensity of light and space environment to further present a different time and the eyes to adapt to the situation of the appropriate adjustment. Effectively improve the brightness of the background and enhance contrast and reflectivity, and many other conditions. To further highlight stereo feeling, administrative levels feeling and art. Effectively promote the commodity aesthetic level.

\section{The beautiful sex of commercial space design principles}

In lighting design ways, in the lights of the media business space environment design not only be able to reflect the functional principle of lamplight, more able to reflect the beautiful sex of lighting design principles. If you want to lighting design becomes an important part of interior space design, it must be able to pay attention to the alignment of lights and commercial space, let the light media gradually become a part of the current, make lighting design to be able to play the role of lighting not only, more want to commercial space into its nostrils the breath of art. In people's real life, we can not only make full use of good lighting design, more should make full use of lighting design in the beautiful sex principle for indoor space form the breath of a culture. Through the space design bold on the decoration and art processing, add on a peculiar modelling beauty for goods. In the light of the indoor space environment, we must be able to properly choose the colors and shapes of lamps and lanterns and light color, let the lamps and lanterns is effectively integrated into the commercial space of the whole environment. Better balance a space layout. Add space shape and space beauty effect. To let the lamps and lanterns of exquisitely beautiful and modern building organically into a whole, for people to create a more beautiful and harmonious aesthetic environment.

\section{Commercial security principles of lighting design in the space}

In modern commercial space, lighting design is a major premise to ensure safety, in some ways, lighting decoration belongs to a form of energy lighting, so, in the process of light exposure, we must be able to consider the safety of lighting decoration, to the use of reasonable safety power supply, avoid the occurrence of safety accidents, because of the complexity of the circuit in the mall, in the design of tube to be able to fully consider the large load of power consumption, and also more light setting number to, therefore, we must consider to the safety of lighting decoration and design scientific, so as to really ensure the safety of the lighting design principles, to ensure the safety of the whole commercial electricity. 


\section{How to use the light media using the technique of the commercial space}

\section{The light the principle of hierarchical design requirements}

On the one hand, we must be able to make good use of hierarchical light media lighting design technique, it is an important means of commercial display at present stage, on the one hand, we must be able to make full use of the environment light environment layered method, this is a not for a specific commodity lighting using method of the target, popular, this is just to provide commercial space in the light, convenient for people walking in the commercial space of light, to be able to satisfy the requirement of the basic visual. For business activities, it's just in order to be able to fully display space itself unique artistic style, therefore, when using this method, must adopt the concealment type lamps and lanterns, especially can make use of compact fluorescent lamp or fluorescent lamp space design, in addition, the use of light layering method at the same time, to be able to use accent lighting method to carry on the design, on the one hand, the accent lighting can be effectively have emphasis and prominent role, highlighting lighting can be used to more effectively express commodity unique features, such as in the display of works of art, the details of the decorations and goods display, etc., can take advantage of these methods, in most cases, this kind of focused lighting method can be adjusted in certain circumstances, for example, mobile on the goods location, in order to be able to better adapt to the commodity of the change the surrounding environment, must be properly adjusted. At the same time, this also is in order to be able to meet the requirements of commercial space visual effect. In light control personnel must pay attention to the environment of the different nature of the workplace, work requirements of lamps and lighting level. Another example, in clothes field, on the choice of light to pay attention to the intensity of light wants downy, do not produce glare, for parking place, is not very tall to the requirement of the light, when the design as long as can meet the demands of daily homework, as far as possible use relatively gentle light.

Similarly, in the hierarchical lighting, we according to the decorative lighting levels, to further absorb the line of sight of people as the main goal, main purpose of doing so is to use a variety of decorative lighting for the space plays the role of the foil. To some extent can also be designed for indoor and environment subject to give plays an important role. On the other hand, business requirements for the decorative lighting in the space environment, are generally follow the following several design principles; The first is to the space that itself of lamps and lanterns in modelling and create a good atmosphere for lighting. Second, is to be able to change in color and light on the light itself has certain adornment effect, finally is lamplight decorate personnel in light space and material on the surface of decorative effect, in addition, still can use special advanced lighting technology bring goods to decorate different visual effect.

\section{Decorative lighting media business forms}

In terms of lighting decoration, lamps and lanterns is using appearance modelling and its decorative lighting, can be in the modelling of lamps and lanterns on the special design, so that you can effectively highlight the characteristics of commercial space, lamps and lanterns in the shape of the past, we can find that this is a kind of historical culture formed by the condensation of fixed artistic style. And at this stage in the appearance of lamps and lanterns, can use modern technology to development of decorative lamps and lanterns, such as from these aspects, such as, special effects to shoot the light and LED neon lights so that you can produce all kinds of shape, can produce unique color, thus can greatly enhance the imagination space in the design of lamps and lanterns, to some extent, the luminous way of lamps and lanterns is not the same, from the traditional manual adjustment to the application of a computer automatic command, so that they can produce the dynamic changes of the color and intensity of illumination and other regular, fantasy decorative effect in the commercial space.

From the perspective on the one hand, the colour of the light itself and flare can produce rich decorative effect, so that they can have a positive impact on people, in the light of light can produce extremely rich rhythm feeling and metrical sense, in the indoor space to produce a perfect "light paintings", to the dynamic effect of the light itself produced bright is dazzing, can effectively constitute a regional center, this is advantageous to the commercial space display advertising, 
multiplicity of transmission can produce light changes, so that you can use in the commercial space, or commercial products can be according to different key expression.

\section{Light media innovations in the commercial space}

In our country commercial lighting design, lighting design is like a spotlight, any object can blossom strange color on the light. Aiming at the present stage lighting design diversification of business environment, must we continuously improve our ability to innovate, especially pay attention to the light and the relationship between the characters, and based on the illuminate of lamplight can form the multi-level and diverse shapes, such as lamps and lanterns can be shaking around on a bigger space, so that the images of light form according to walking around and form a good interactive relationship, thus to move around in a space, smooth shadow and it can gain more interest in commercial space, also can fully reflect light flow in the application of environmental art, this is mainly due to both from the economic efficiency and service life of lamps and lanterns is beautiful on both benefits greater than traditional lamps and lanterns, also more artistic effect.

\section{Treatment of space and light}

In general, the space and light between the two can form a good interactive relationship. First of all, is the gala lighting environment, for gala environment, light environment is the space of a wide variety of lighting, can form has obvious randomness and colorful, through a variety of light source can be effectively made with flash effect, in the cheerful lighting environment, the environment is often reflected in the larger foot path or a commercial pedestrian street of less than, can set up some relatively weak, moderate light, so that it can effectively relieve people's psychological burden, give a person a kind of relaxed feeling.

In addition, we also can choose a few relatively simple cautious lighting environment, this kind of lighting environment are generally use of people's attention, provide people with a good visual environment, which can make people blundering heart become more quiet, no obvious contrast in lighting arrangement.

Conclusion: to some extent, can light media art for the current business environment to build a good environment of art, let the light of the visual art movement gradually enriched the contents of the space environment of environment effectively, not only can effectively meet the demand of the current commercial lighting, and more importantly provide comfortable environment for people, based on the characteristic of light media in commercial space requirements, in view of the current lighting use principle analysis, put forward how to skillfully use light media in the use of commercial space, hope to be able to better help the light media in use and development of commercial space.

\section{References}

[1] Liu Jinxia jian-qun Lin. Analyze the interior business scale in space [J]. Journal of art and design (theory). 2007 (7)

[2] GuanShuGuang. Analyses the modern commercial space display design [J]. Journal of beauty and age. 2007 (9)

Qiu Yu [3]. The design of commercial space program [J]. Journal of anhui literature (second half). 2007 (12)

[4] our fan. When city met art expression in shallow theory of transparency in commercial space [J]. Journal of Shanghai business. 2009 (05)

[5] Yuan Jincheng LuoChao, Zhu Xiang. Green exhibition in the application of modern commercial space research in China $[\mathrm{J}]$. Journal of art research. 2010 
Author introduction: on January 2, 1982 was born, female, han ethnic group, shandong j, bachelor degree, teaching assistant, architectural decoration 